\title{
Gary Liney, Uulke van der Heide (Eds). MRI for Radiotherapy. Planning, Delivery, and Response assessment
}

\author{
Springer Nature Switzerland AG, 2019, ISBN 978-3-030-14442-5
}

\author{
Cesare Guida $^{1} \cdot$ Valerio Nardone $^{1}$ \\ Published online: 2 February 2021 \\ (C) The Author(s), under exclusive licence to Springer-Verlag GmbH, DE part of Springer Nature 2021
}

This book is the first edition of the volume published in 2019 by Gary Liney and Uulke van der Heide.

G. Liney, one of the major experts in the use and integration of MRI techniques into radiotherapy planning, is the senior medical physicist at the Ingham Institute for Applied Medical Research and the Liverpool Cancer Therapy Centre in Sydney, Australia. U. van der Heide works as a medical physicist at the Netherlands Cancer Institute (NCI) in Amsterdam, the Netherlands. He has been the director of the ESTRO "Imaging for Physicists" course until 2017 and leads the MR-guided radiotherapy program at the NCI. Many renowned experts in the multiple disciplines involved in the field, such as physics, radiology and oncology, have been invited to treat the different issues.

Although the first applications of MRI in radiotherapy were started since the 1980s, a growing interest has been stimulated only in the last years, mainly because of the exciting developments achievable by hybrid MRI treatment devices. In particular, in 2017, while the first patients started to be treated on commercial MRI-Linac systems, the long-term follow-up results of patients analysed with the early pioneering MRIcobalt machines, showing the benefits of the hybrid approach, were positively recognized.

The relevance of this publication derives from the completeness of the editorial approach, which considers, at our knowledge for the first time, all the aspects involved in an overall way, regards to the application of MRI in radiotherapy, from physics to the clinical prospective. Being each chapter highly specialized and updated to answer to specific queries, the lecture and the study of the whole book is favoured by a

Cesare Guida

cesareguida@gmail.com

1 Ospedale del Mare, ASL Napoli 1 Centro, Naples, Italy logical progression from simulation through to the real-time guidance.

Structured in two hundred pages, full of images and tables, the publication is organized in five parts, divided into thirteen chapters. More precisely, Part I (MRI for Planning) contains the following chapters: (1) Implementation and Acquisition Protocol, (2) MRI to CT Image Registration, (3) Quality Assurance and (4) Clinical Application of MRI in Radiotherapy Planning. Part II (MRI During Treatment) includes the following chapters: (5) Functional MR Imaging, (6) Response Assessment and (7) Motion Management. In Part III (MRI-Only Radiotherapy) are treated chapters: (8) Challenges and Requirements and (9) MR-Only Methodology. Part IV (MRI for Guidance) contains the following chapters: (10) MRI Linac Systems and (11) MRI at the Time of External Beam Treatment. Part V (Future Direction) includes the following chapters: (12) Will We Still Need Radiotherapy in 20 Years? and (13) Real-Time MRI-Guided Particle Therapy.

As it can be clearly understood from the above contents, this publication treats all the workflow of MRI applications in a radiotherapy department, as well as future directions of hybrid devices.

Being the first unified publication on MRI in radiotherapy, this book widely deserves a prominent position in libraries and on work desks in radiotherapy departments, as we are sure that this approach will be broadly applied in the next future. Furthermore, we suggest this volume to residents in radiotherapy and medical physics, as well as to those studying radiology and oncology. At the same time, the book is aimed and suitable also for a wide audience of clinicians, with main reference to those operating in diagnostic imaging and oncology.

Publisher's note Springer Nature remains neutral with regard to jurisdictional claims in published maps and institutional affiliations. 